the intemperate statements either of supporters or of critics. Nor be it thought that in the sphere of treatment Koch's outlook was limited to tuberculin and its applications. He approached the consideration of treatment from many angles. His incessant labours pointed the way to most subsequent developments in the direction of active and passive immunization, and no less to the everrecurring search for chemotherapeutic agents. The investigators in tuberculosis are few who have not sometimes been surprised by evidence-in whatever corner of the field their research-that Koch had paced the ground before them.

I think it may surely be said without fear of dissent that in few, if in any, departments of medicine has there occurred during fifty years so complete an upturn of thought and practice as in that of tuberculosis. The credit for this is primarily due to Koch. To him we owe it that knowledge has replaced ignorance, that doubt and helplessness have largely disappeared, that preventive measures and therapeutic procedure are now inspired and controlled by ascertained fact, that the morbidity of tuberculosis has lessened, and that in all progressive countries the mortality from tuberculosis is tumbling rapidly. Robert Koch laid the solid foundations on which the rest has been built, and to him be the glory.

\section{A British Medical Association Lecture} ov

\section{NASAL SINUS INFECTION*}

WALTER HOWARTH, M.A., M.B., F.R.C.S.

SURGEON TO THE EAR, NOSE, AND THROAT DEPARTMENT, ST. THOMAS'S HOSPITAL； PRESIDENT OF THE LARYNGOLOGICAL SECTION, ROYAL SOCIETY OF MIEDICINE.

The nasal accessory sinuses are lined, like the nasal cavity itself, by a ciliated mucous membrane which, however, is not so rich in mucus-secreting glands. The action of the cilia, which work upwards towards the ostia, normally keeps the sinus free from secretion.

The reactions of the mucous membrane to infections have recently been the subject of research, and it is perhaps legitimate to conclude that retention of mucus or of pus in a sinus is an indication that the cilia have failed in their attempt to remove this, owing to damage to, or destruction of, the ciliary mechanism. The aim of surgery in treating sinusitis is to remove the discharges from the mucous membrane in order that it may be rested, and may utilize such power of spontaneous recovery of function as it possesses. What, then, are the infections that attack the nose, and why should such infections involve the accessory sinuses? It is being more and more clearly recognized that mucuslined cavities are subject to catarrhal and infective inflammation, somewhat in proportion to the degree of obstruction to their drainage and ventilation. Thus it is in nasal cavities that show some deviation from the normal that nasal sinus infection is more likely to occur. It is certainly unusual for these complications to occur in a perfectly healthy nose unless the original infection is a very virulent one or the resistance of the individual is for some reason considerably lowered. The predisposition of individuals to upper respiratory tract infections has been shown to be increased when vitamin $\mathrm{A}$ is deficient in the diet, whilst a lack of endocrine balance, and occasionally some form of allergy, are other factors that lay the mucous membrane open to infection.

* Read before the Chesterfield Branch on March 11th.
There seems to be no doubt that influenza is the most potent factor in the causation of sinus affections, though any organism that can cause an acute coryza (and I include here those organisms associated with the specific fevers) may, if conditions are favourable, produce sinus complications.

\section{Abnormalities Predisposing to Sinusitis}

It may be well, for a moment, to turn our attention to a consideration of the nasal passages and some of the common abnormalities that are met with there. There is no need to remind you that the outer wall of the nose is its most important part, because it is under the middle turbinate that the anterior group of sinuses have their openings. The frontal sinus lies most anteriorly, then come the anterior ethmoidal cells, the maxillary antrum further back, whilst above the middle turbinate the posterior ethmoidal cells and the sphenoidal sinus open into the spheno-ethmoidal recess. Consequently all forms of nasal obstruction, either by actual pressure or by inducing congestion, may interfere with the drainage from these sinuses or affect their aeration, and so render them more sensitive to the spread of infection from the nasal passages.

A deviation of the septum, whether traumatic or otherwise, naturally plays a large part in causing obstruction in the middle turbinate region. This is the most serious form of obstruction, as it causes more severe inflammatory disease of the accessory sinuses than does obstruction elsewhere, for such a deformity may interfere not only with the drainage and ventilation of the anterior group of sinuses, but also, by blocking the olfactory fissure, may impair that of the posterior ethmoidal cells and sphenoidal sinuses as well. Besides high deviations of the septum there are other conditions which may cause obstruction in the middle turbinate region.

We have hypertrophic conditions of the middle turbinate itself and extra air cells in the middle turbinate, which is merely a folded-over portion of the ethmoidal labyrinth. These may close the olfactory fissure, or press unduly on the diatus semilunaris. Then again, an enlarged bulla ethmoidalis may obstruct the hiatus semilunaris and disturb its contour, thus interfering with the ventilation and drainage of the anterior group of sinuses. Finally, the infundibulum itself is often surrounded by ethmoidal cells, and an extra large one in the region of the uncinate process may block the infundibulum and so cause a predisposition to infection and inflammation.

The conditions mentioned are by no means unusual, and when combined with the appropriate pathogenic microorganisms and a lowered resistance of the individual, it is scarcely to be wondered at that nasal sinus infection is so commonly met with.

\section{Examination of a Typical Case}

It will now be well to consider an acute infection of nasal origin and its mode of spread to the sinuses.

When infection occurs $I$ think it is true to say that the maxillary antrum is always involved in the process. Though the antral condition may often subside and leave some other sinus more prominently involved, in the initial stages it seldom, if ever, escapes. When, during a common cold, the symptoms such as headache, pain, tenderness, unilateral nasal discharge, redness or swelling of the face lead one to suspect a sinus infection, a careful nasal examination must at once be undertaken. The mucous membrane will be found to be intensely engorged. Introduction of a pledget of cotton-wool wrung out of 20 per cent. cocaine will not only mop up any secretion, but will also cause the inflamed mucosa to shrink, and make 
inspection of the middle meatus of the nose easier. Pus may be seen in this cleft either anteriorly or far back in the nose. When this is mopped away a further pledget of cotton-wool with cocaine should, if possible, be inserted right under the middle turbinate to shrink up the mucosa in the region of the hiatus semilunaris, and enable one to come to some conclusion as to the origin of the pus. When the pus is seen far forward, near the anterior end of the middle turbinate, its origin is probably from the frontal sinus or ethmoidal cells, particularly if it reappears quickly after being mopped away. If it is seen far back in the cleft, and if some alteration in the position of the head, such as putting it between the knees and turning the affected side uppermost, causes it to reappear, then this occurrence is evidence that the pus comes from the maxillary antrum, as the altered position of the head places the ostium of this sinus in a situation more suitable for drainage than that afforded by the upright posture.

\section{Further Steps in Diagnosis}

It is important for us to ascertain definitely what sinus is involved, whether the maxillary antrum alone is affected, or whether there may be some involvement of the frontal and ethmoidal sinus as well. It is, I think, true to say that when the frontal sinus and ethmoid are involved the antrum is always affected, but the converse does not hold good. We can obtain direct proof of the presence of pus in. the maxillary antrum by puncturing it and sucking out or washing out the contents. This relatively simple procedure is best carried out under the inferior turbinate. I find a Coakley trocar and cannula the most efficient instrument for this purpose.

When this proof puncture is positive we have established the fact that the antrum is infected, but we have not determined whether it alone is involved or whether it is acting as a reservoir for pus which has been secreted in one of the sinuses higher up-the frontal sinus or the anterior ethmoidal cells. To ascertain this it is really necessary to wait some little time and then see whether pus again appears under the middle turbinate. This inspection is facilitated by the introduction of a Killian speculum beneath the turbinate after cocainization. It has been said that it is possible to introduce a Hartmann's cannula into the frontal sinus and aspirate it, but in acute cases my experience is that the attempt is too painful and difficult, on account of the swollen mucosa, and should not be tried. Still more do I condemn, for diagnostic purposes, the proposal that a portion of the turbinate should be resected in order to facilitate the passage of a cannula.

It is customary to try to obtain confirmatory evidence of sinus infection by transilluminating the cavities, and though in some cases it may be of use, it is apt to be fallacious, more particularly in the acute conditions. A good $x$-ray film taken from a proper angle is a more useful help.

\section{Treatment in Anterior Sinus Infection}

When it is established that one or more of the anterior group of sinuses is affected, what should be our line of treatment? This should be both general and local. General treatment consists in ordering the patient to bed, more particularly if the headache and general disturbances are severe, and giving broken doses of calomel every hour until the bowels are well open. The leucodescent head bath, if available, should be applied, and 15 grains of aspirin given to cause diaphoresis. Local treatment consists in the careful application to the middle meatus of a 20 per cent. solution of cocaine on cotton-wool at least twice a day. This is, to my mind, the most valuable procedure that the surgeon can adopt, as by its use the mucosa of this region is rendered ischaemic and the drainage from the sinuses markedly facilitated. Douching with very hot saline solutions after this will further relieve the congestion of the mucosa. In the interval, steam inhalations of menthol in friars' balsam every three or four hours is a most useful help in facilitating the discharge of inflammatory products from the sinus by shrinking the mucosa and stimulating ciliary action. Phenalgin in 7-grain doses may be of value in relieving the headache.

The large majority of cases undergo resolution by these means, more particularly if the local application of cocaine by the surgeon is efficiently carried out. Some surgeons rely on more frequent puncture and lavage of the antrum. In my opinion this is seldom necessary after the first aspiration, and, if efficient drainage can be assured by other means, antral irrigation should be dispensed with. In very severe cases it may be justifiable to perform it four or five times at three-day intervals. After the antrum has been emptied it is better to use an oily solution, such as liquid paraffin containing $1 / 4$ per cent. phenol, in preference to the watery solutions usually recommended. For many years $I$ have used $1 / 4$ per cent. phenol in an American preparation called benzoinaquol, but this is no longer available.

So far we have considered the spread of infection to the anterior group of sinuses as occurring by extension from the nasal cavity, but it must be remembered that the maxillary antrum may be infected by the spread of infection from the roots of teeth that are in contact with the antral cavity. This route of infection is responsible for about 20 per cent. of all cases of maxillary antritis. There is no need to mention in detail the causes of dental infection, but apical abscesses, faulty extractions, and periostitis, when the second premolar and first and second molars are involved, are among the most common. The antrum in these cases of dental origin is apt to contain very foulsmelling pus, but usually clears up after intranasal treatment and aspiration. Two or three washings-out with an oily solution may be necessary. It goes without saying that the tooth which is the cause of the infection should be removed.

Although in a large majority of cases satisfactory drainage occurs, or can be promoted through the ordinary channels, there are cases in which from the outset the normal ostium becomes occluded and the products of inflammation remain locked up in the sinus. In these cases the symptoms may be extremely severe and the inflammation show itself externally by swelling. When the maxillary antrum is the site of a closed empyema there may not only be a bulging of the naso-antral wall in the region of the meatus, but there may also be a swelling under the upper lip (in the labio-gingival fold) if the abscess has burst through the anterior. wall of the antrum in the region of the canine fossa, where the bone is thinnest. In these cases the measures to be adopted should be as conservative as possible, but it will not be possible to avoid opening the abscess under the lip and making a proper counter-opening into the nose through the nasoantral wall. If this is done the inflammation should rapidly subside.

Acute empyema in the frontal sinus and in the anterior ethmoid may produce symptoms such as intense pain in the forehead, with redness, swelling, and oedema in the region of the inner canthus and upper eyelid; there may even be swelling over the frontal bone if acute osteomyelitis has occurred. It may not be easy to determine the origin of the swelling, but it may be said that when the frontal sinus is the source the swelling and tenderness is apt to be near the inner canthus over the inferior wall of the sinus, whilst if the ethmoid is responsible the 
swelling is usually lower down in the region of the lachrymal sac, and may, indeed, be mistaken for a lachrymal abscess. It is a difficult matter to decide whether a case with swelling and oedema of the eyelid and in the region of the inner canthus should be treated conservatively by antral puncture, and possibly by removal of the anterior end of the middle turbinate, or whether an external incision should at once be made. A good many cases, particularly in children, where there is some oedema of the eyelid and swelling in the lachrymal region, undoubtedly settle down when purely intranasal conservative measures are employed, but no hard-and-fast rule can be laid down, as each case must be judged on its merits. Suffice it to say that in those cases in which the infection is particularly acute, where the onset of external symptoms is rapid, and where the toxaemia is severe, I think it essential that an external incision should be made, and that the surgeon who withheld it would submit the patient to a risk of orbital cellulitis, meningitis, or other serious complication.

In no case, however, should any elaborate operation be attempted, and the surgeon should content himself with evacuating the pus, inserting a rubber drain, and leaving the wound open in its lowest part. It is, however, of the utmost importance that a good counter-opening should be made through the ethmoid labyrinth into the nasal cavity, so that the main drainage may take place this way. If, after twenty-four hours, resolution of inflammation appears to be taking place, the rubber drain in the external wound may be removed, and it can then be allowed to close. If the external swelling appears over the anterior wall of the frontal sinus the bone should not be interfered with for, fear of setting up osteomyelitis ; but if this complication has already occurred, as is likely to happen if the abscess has burst through the diploic bone, removal of the bone is a paramount necessity, and this should be extended until healthy bone is reached.

\section{Involvement of Posterior Sinuses}

Acute infection of the posterior group of sinuses is a much rarer complication of nasal disease, and is more difficult to recognize. If we recall the position of the ostia of the posterior ethmoidal cells and of the sphenoidal sinus as they open into the spheno-ethmoidal recess, it is clear that secretions from these sinuses must appear either in the olfactory fissure between the septum and the middle turbinate, or posteriorly in the choana above the middle turbinate, or on the lateral wall of the nasopharynx. In this situation the discharge can only be seen by posterior rhinoscopy with the mirror-not always an easy procedure. The inspection of the olfactory fissure from the front may be facilitated by the use of the longbladed Killian speculum, which presses the middle turbinate out of the way and affords a more direct inspection of the anterior wall of the sphenoidal sinus, and permits the insertion of a trocar and cannula into it. In this region the careful use of cocaine is again of the utmost value.

The symptoms that are associated with acute infection of the posterior group of sinuses are somewhat equivocal, but in the case of the sphenoidal sinus the headache is usually severe, and tends to be localized to the parietal and temporal regions, often radiating to the ears. A curious discomfort is often felt in the ears themselves, and ocular symptoms, particularly tenderness of the eyeball, are often marked. General symptoms, such as fever, sleeplessness, giddiness, and malaise are more prominent than is the case in an ordinary cold, and these symptoms tend to get worse instead of better.

Treatment should be on the lines indicated for the anterior group to ensure as free drainage as is possible, but when the cocaine-bearing cotton-wool is inserted by the surgeon it should be placed between the septum and the middle turbinate to keep the olfactory fissure as widely open as possible. It may be necessary to insert a trocar and cannula into the sphenoidal sinus and suck out the secretion. Access to the anterior wall of the sphenoid is not always easy, but in a considerable number of cases it can be reached, especially if a long-bladed Killian speculum is used. It may, however, be necessary to give the patient some gas and oxygen, as this region of the nose is difficult to anaesthetize locally, particularly if the mucosa is in an inflamed condition. In some cases where the septum is markedly deflected it may be necessary to resect a portion of the middle turbinate.

In the treatment of all these cases of acute nasal sinus infection our aim is to promote the resolution of inflammation as soon as possible and to establish immunity. It has recently been suggested that when any toxic process is present in the body there is a tendency to acidosis, and that this acidosis demands compensation from the bases circulating in the blood or in the tissues, which are no longer available to play their part in the process of immunity. This affords some explanation of the fact that the administration of sufficient sodium bicarbonate to keep the urine alkaline will often help to promote the resolution of inflammation and bring about a more speedy cure.

\section{Chronic Sinusitis}

The consideration of nasal sinus infection in its more chronic form opens so large a field that it will not be possible to do more than touch upon it. We have seen that the sinuses when affected acutely show a marked tendency to resolution, but in some cases the infection overcomes this tendency and the disease becomes chronic. Among the causes which may give rise to this are: (1) conditions which interfere with normal drainage ; $(2)$ excessive virulence of the infecting micro-organisms; (3) repeated attacks of inflammation; (4) the presence of inflammatory changes in the mucous membrane ; (5) secretion flowing in from another sinus ; and, lastly, the susceptibility of the individual.

Patients suffering from a chronic nasal sinus infection usually come under observation on account of headache, nasal obstruction, discharge, or the complaint of a chronic cold in the head. On the other hand, many cases may be discovered in the search for a septic focus that is now conducted with such vigour in an attempt to explain digestive, respiratory, ocular, or other phenomena. It is therefore of the utmost importance that we should be able to deal adequately with these chronic conditions. In some ways recognition of sinus infection in its chronic form is easier than when it is acute, in that the nasal passages are less severely congested and examination is therefore less difficult.

The detection of pus under or above the middle turbinate or in the post-nasal space, the presence of polypi in the nostril or in the choana, crusting in the nose or nasopharynx, or secondary changes in the larynx will help to guide us towards the group of sinuses that is affected, and it should not be very difficult, with careful examination, $x$-ray photography, proof puncture, and suction to get a reasonable idea of the extent of the infection and its exact situation. When suppuration is well established in any sinus it is, to my mind, rather a waste of time to devote much attention to attempted relief by puncture and lavage, and treatment should, I think, be mainly surgical. I am not in favour of drastic surgical measures for the cure of suppuration in any sinus, and think that if steps are taken to provide adequate drainage the vast majority of cases will settle down satisfactorily, but the drainage provided by the operation must be sufficient to drain the cavity completely. Surgery abhors dead spaces, and nowhere more so than in the sinuses of the nose. 
Details of Local and General Treatment

For the maxillary antrum the purely intranasal opening under the inferior turbinate, if of sufficient size, will usually suffice, and in this connexion I should like to say that the inferior turbinate should be left intact, or only the smallest portion of its lower border near the anterior end removed. I seldom find it necessary to resort to the Caldwell-Luc operation, and I actively dislike the Denker and Canfield operations, as I can see no reason for the destruction of the anterior angle that these operations seek to encompass. I regard the treatment after the intranasal operation as of importance. It may be summed up in the word-inactivity. In other words, I do not believe in washing out the cavity, but prefer it to drain naturally through the opening provided. If the discharge does not diminish with sufficient rapidity, and it is thought that lavage might help, the solution used should be an oily one. General tonic treatment and the use of sufficient alkalis to keep the urine alkaline will help the resolution of inflammation.

In recent years the question of chronic infection of the ethmoidal labyrinth has received a great deal of attention, and our views about it have undergone alterations that have somewhat disturbed previously accepted ideas. I am accustomed to recognize two forms:

1. The hyperplastic type with polypus formation.

2. The suppurative type.

As both types vary in extent and in severity, so, naturally, does the treatment required for their relief. The basic principles of aeration and drainage for the successful treatment of all sinus infection, however, hold here as elsewhere, and I regard it as important to remove the middle turbinate, more particularly the high anterior part that overhangs the infundibulum, and thus to free the approach to the cells. In mild cases the prolonged application of tampons of 10 per cent. argyrol in glycerin may effect a cure in hyperplastic cases, whilst in others the whole polypus-bearing area will require to be removed. The suppurative cases, again, vary in degree, and so does the extent of treatment. Sometimes after removal of the middle turbinate a very limited removal of particular cells will effect a cure, whilst in others the complete exenteration of the whole labyrinth by intranasal operation, or even by the approach afforded by an external incision, may be necessary.

Suppuration in the frontal sinus presents a variety of problems and an infinity of possible solutions to them: Our endeavour should be to provide the aeration and drainage, and then to be as conservative as possible, always remembering, however, that in the vast majority of cases the anterior ethmoidal cells are also involved, and that the treatment of the ethmoid is the key to the frontal sinus.

As regards the variety of methods of intranasal approach I will only say that they demand considerable experience and some operative skill. I am not in favour of cutting or rasping the fronto-nasal duct, owing to the tendency to new bone formation and recurrence of obstruction. I believe that if the region of the infundibulum can be cleared of cells and the approach to the ethmoid freed, the dilatation with metallic sounds will often be sufficient to effect a cure. In intractable cases in which an external operation seems to be indicated I prefer the operation that I described many years ago, and which goes by my name, as it seems to me to provide adequate drainage whilst preserving the lining mucosa. Moreover, this is accompanied by no deformity, and by a scar which is practically invisible.

Chronic infection in the posterior group of sinuses is by no means easy to diagnose, and usually comes under observation owing to descending infections manifesting themselves in the digestive or respiratory tract, or on account of eye symptoms, such as retrobulbar neuritis, optic atrophy, or oculomotor phenomena. General toxic symptoms, or intense occipital or parietal headache, may also demand investigation, whilst local symptoms, such as dryness and crusting of the pharynx or secondary laryngeal inflammation, may be found to be of post-nasal origin. The treatment of the posterior group consists in opening them as freely as possible and allowing them to drain. The middle turbinate should be resected and the large posterior ethmoidal cells entered. It should be remembered that the most posterior ethmoidal cell is, as it were, stuck on to the outer part of the anterior wall of the sphenoidal sinus, and that lateral to this junction is the dangerous area of the cavernous sinus. The anterior wall of the sphenoidal sinus is readily broken down, and free drainage and aeration thereby secured.

\section{ATEBRIN IN THE TREATMENT OF SEVERE MALARIA BY}

A. J. ORENSTEIN, C.M.G., LL.D., M.D.

(From the Johannesburg General Hospital, Department for Tropical Diseases.)

The following is merely a brief report of the results obtained with atebrin (until recently known as erion) in the treatment of eight cases of severe malaria in hospital. The number of cases is too small to admit of any definite conclusions being drawn, but the work was interrupted by my departure to Europe. It seemed, however, that in the early stages of experience with a new remedy reports even of a few cases would be of some value.

The cases here recorded were deliberately chosen for the following reasons. They gave positive blood findings ; they were severe-two very severe-cases; and they were residents of Johannesburg, where there is no endemic malaria-their subsequent history in respect of recrudescences, therefore, would be clear-cut. The plan of treatment was the same in all cases. One decigram of atebrin and one centigram of plasmoquine were given three times a day for four successive days; after a few days the course was repeated.

The plus signs on the temperature charts in connexion with the blood findings have the following significance: $+++=$ numerous parasites in every field in a thick drop preparation $;++=$ numerous parasites; and $+=$ para . sites seen in most fields. The original charts were fourhourly, but the reproductions have been reduced to a twice-daily basis, and terminate when the temperature settled down to normal. All the blood examinations were done personally by me. Giemsa stain was used.

Up to the time of leaving Johannesburg (March 10th) none of the patients treated had relapsed: One patient now residing in London, who was severely ill, reported recently as being in perfect health ever since the treatment, which terminated early in January-that is, about four months ago. Slight scleral jaundice was observed in one or two cases, but it is uncertain whether this was due to the drug or to the malaria. No unpleasant symptoms due to atebrin were observed, but in three cases there were the headache and cramps one sees occasionally in patients somewhat intolerant to plasmoquine. On the whole, I am most favourably impressed with the value of atebrin.

\section{CASE I}

A male native, aged about 19 years, was admitted on September 26th, 1931, with a history of several previous attacks of malaria. Present attack: first rigor on day of admission; spleen enlarged and palpable. Atebrin and plasmoquine were started on September 29th and repeated 